\title{
Naturalness and chaotic inflation in supergravity from massive vector multiplets
}

\author{
Fotis Farakos and Rikard von Unge \\ Institute for Theoretical Physics, Masaryk University, \\ Kotlarska 2, 61137 Brno, Czech Republic \\ E-mail: fotisf@mail.muni.cz, unge@physics.muni.cz
}

ABSTRACT: We study the embedding of the quadratic model of chaotic inflation into the $4 \mathrm{D}, \mathrm{N}=1$ minimal theories of supergravity by the use of massive vector multiplets and investigate its robustness against higher order corrections. In particular, we investigate the criterion of technical naturalness for the inflaton potential. In the framework of the newminimal formulation the massive vector multiplet is built in terms of a real linear multiplet coupled to a vector multiplet via the $4 \mathrm{D}$ analog of the Green-Schwarz term. This theory gives rise to a single-field quadratic model of chaotic inflation, which is protected by an shift symmetry which naturally suppresses the higher order corrections. The embedding in the old-minimal formulation is again achieved in terms of a massive vector multiplet and also gives rise to single-field inflation. Nevertheless in this case there is no obvious symmetry to protect the model from higher order corrections.

KeYwords: Cosmology of Theories beyond the SM, Supergravity Models

ARXiv EPRINT: 1404.3739 


\section{Contents}

1 Introduction $\quad 1$

2 Chaotic inflation in new-minimal supergravity 2

$\begin{array}{lll}3 & \text { Chaotic inflation in old-minimal supergravity } & 7\end{array}$

$\begin{array}{lll}4 & \text { Conclusions } & 10\end{array}$

\section{Introduction}

There has been stimulating progress in observational inflationary cosmology. The BICEP2 experiment [1] has announced the observation of the ratio of tensor to scalar perturbations of the metric to be

$$
r=0.2_{-0.05}^{+0.07}
$$

which has triggered a discussion [2-7] of the implications of these findings on theoretical models for physics beyond the electroweak scale.

The simplest model which is favored by the combined PLANCK and BICEP2 $[1,8]$ data is the quadratic model of chaotic inflation [9], with potential

$$
\mathcal{V}=\frac{1}{2} m^{2} \phi^{2}
$$

which for 50 e-foldings of inflation gives rise to a ratio of tensor to scalar perturbation of $r \approx 0.16$.

The new experimental results and the fact that inflation is a high energy process gives us a new possibility to study models of physics beyond the Standard Model. In particular, any serious candidate for new physics will have to be able to reproduce these results. In this work we will focus on supersymmetric theories. It is then important to embed the chaotic model of inflation (1.2) into the theory of supergravity [10, 11].

An embedding into old-minimal supergravity was carried out successfully in ref. [12], by employing chiral multiplets and was further discussed in ref. [13]. A general chaotic inflation was also discussed in ref. $[14,15]$, where these proposals are easier to embed into phenomenological models. An interesting attempt to give a geometric origin to a chaotic inflationary phase in supergravity was initiated recently in ref. [16], again involving two chiral multiplets in the dual picture, and further discussed in ref. [17-19].

Another interesting possibility is to study models with massive vector multiplets, first coupled to the old-minimal supergravity in ref. [20,21], where also the quadratic potential was initially discussed [21]. The relation of massive vector multiplets to new-minimal higher 
derivative supergravity was first pointed out in ref. [22]. In a series of papers [23-25] singlefield inflationary models utilizing massive vector multiplets and possible higher derivative corrections were systematically studied, and a single-field chaotic model was introduced in ref. [24]. Moreover, the gauged isometries of the minimal supergravity models of inflation were investigated in ref. [26, 27]. Quadratic chaotic inflationary models where the D-term dominates over the F-term were studied in ref. [28, 29]. Finally, a different perspective on chaotic inflation from D-terms in supergravity may be found in ref. [30].

In general, the potential (1.2) is not straightforward to reproduce. The usual issues one encounters are

- Identify the one and only scalar which drives inflation.

- Stabilize the other scalars, and explain why they do not ruin inflation.

- Higher order corrections may spoil inflation; the notorious $\eta$-problem.

In this work we investigate the possibility of embedding the quadratic model of inflation in supergravity, with the use of massive vector multiplets and we will see how the aforementioned issues are addressed. In particular, a generic property of inflationary models utilizing massive vector multiplets is that there is no need to stabilize any additional fields nor identify the inflaton; these are by construction single-field models, and thus the first two aforementioned issues are automatically solved. To address the issue of higher order corrections we will rely on the existence of a softly broken shift symmetry and invoke technical naturalness in the sense of 't Hooft [31].

\section{Chaotic inflation in new-minimal supergravity}

Let us start with a real linear multiplet, and couple it to the new-minimal supergravity [3235]. The definition of the real linear superfield in this framework is

$$
\nabla^{2} L=\bar{\nabla}^{2} L=0 .
$$

The definitions of the bosonic components are

$$
L\left|=\phi \quad, \quad-\frac{1}{2}\left[\nabla_{\alpha}, \bar{\nabla}_{\dot{\alpha}}\right] L\right|=h_{\alpha \dot{\alpha}}
$$

with

$$
h_{m}=-\frac{1}{2} \epsilon_{m n r s} \partial^{n} b^{r s}-2 \phi H_{m}
$$

where $b_{m n}$ is the two form of the real linear multiplet and $H_{m}$ is an auxiliary field of the new-minimal supergravity formulation which we will review later. It is easy to verify that the minimal kinematic term

$$
-\int d^{4} \theta E L^{2}=-\frac{1}{2} e \partial \phi \partial \phi+\frac{1}{2} e h_{m} h^{m}
$$

allows a shift symmetry for the superfield

$$
L \rightarrow L+c M_{P}
$$


for some real constant $c$, which translates into

$$
\phi \rightarrow \phi+c M_{P}
$$

for the real scalar lowest component. We would like to stress that the shift (2.5) is also a symmetry of (2.1) and thus does not violate the definition of the real linear multiplet.

The shift symmetry (2.5) protects the minimal kinematic term from higher order corrections and will later shield the model against the $\eta$-problem. For example the possible higher order correction

$$
-\frac{1}{(\text { some scale })^{4}} \int d^{4} \theta E L^{4} \rightarrow \text { violates symmetry }
$$

is ruled out since the quartic term $\left(L^{4}\right)$ violates the shift symmetry.

Of course, this exact shift symmetry (2.5) rules out the possibility of introducing a potential for the theory. Here is where the Green-Schwarz term comes in. It is well established that a gauge anomaly can be canceled by introducing a two-form which couples to the gauge field and gives rise to tree diagrams that cancel the anomalous loop diagrams. This mechanism exists also in supergravity theories and in four dimensions the coupling of the two-form with the gauge field is given by the gauge invariant contact term

$$
-g M \int d^{4} \theta E L V=-\frac{1}{2} e g M \phi \mathrm{D}+\frac{1}{2} e g M v_{m}\left(h^{m}+2 \phi H^{m}\right)
$$

where $V$ the vector superfield of the would-be-anomalous $\mathrm{U}(1)$, and $L$ a real linear multiplet containing the two-form. The fields $\mathrm{D}$ and $v_{m}$ are the auxiliary real scalar and the physical vector of the $\mathrm{U}(1)$ vector superfield. In ref. [36] it was shown that the effect of this term is to introduce a realization of the Stueckelberg mechanism where the two-form of the real linear is eaten by the vector. The Green-Schwarz mechanism was further investigated in minimal supergravity in ref. [37]. It is in fact a way to write down massive vector multiplets [36, 38]. On the other hand this same term (2.7) violates the shift symmetry (2.5) and as we will see it creates a potential. Thus the small breaking of the shift symmetry is generated by the Green-Schwarz term and it is expected that as long as

$$
g M \ll H
$$

where $H$ is the Hubble constant, the effect of the small breaking on the kinematic term (2.4) is negligible. This is natural in the 't Hooft sense since for $g M \rightarrow 0$ the shift symmetry is restored, and the symmetry of the system is enhanced [31]. To better understand this argument it is convenient to think of the mass $M$ as parameterizing the flow of the theory through some parameter space. When the theory sits on the $M_{*}=0$ point, all the operators that violate the symmetry vanish. Far away from the special point $M_{*}=0$, the symmetry violating operators become large. Thus, for a small value of $M$ close to $M_{*}=0$ the operators that violate the shift symmetry have to be highly suppressed. More specifically for example

$$
-\frac{1}{(\text { some scale })^{4}} \int d^{4} \theta E L^{4} \rightarrow \text { naturally suppressed. }
$$


For similar considerations in the old-minimal supergravity framework, in a model of two chiral multiplets, see ref. [12].

Taking this into account we may proceed to investigate the model in more detail. As we have mentioned, our interest now lies in the new-minimal supergravity [32], which originates from the superconformal supergravity after appropriate gauge fixing [39-43]. Note that the new-minimal supergravity allowes only R-invariant Lagrangians. The bosonic sector of the pure theory reads

$$
-2 \int d^{4} \theta E V_{\mathrm{R}}=\frac{1}{2} e\left(R+6 H_{m} H^{m}\right)+2 e A_{m}^{-} H^{m} .
$$

On top of the graviton $e_{m}^{a}$ and the gravitino $\psi_{m}^{\alpha}$, this theory contains two auxiliary fields: the $A_{m}$ which gauges the R-symmetry and the two-form $B_{m n}$, which only appears through the dual of its field strength $H^{m}$. Let us mention that

$$
A_{m}^{-}=A_{m}-3 H_{m}
$$

The properties of this minimal supergravity were investigated in a series of papers $[22,32-$ $36,40,44-47]$.

For the total Lagrangian to be manifestly supersymmetric we will write it down in new-minimal supergravity superspace [34], and then turn to component form. The theory we wish to consider is

$$
\begin{aligned}
\mathcal{L}= & -2 M_{P}^{2} \int d^{4} \theta E V_{\mathrm{R}}+\frac{1}{4}\left[\int d^{2} \theta \mathcal{E} W^{2}(V)+\text { h.c. }\right] \\
& -g M \int d^{4} \theta E L V-\int d^{4} \theta E L^{2}
\end{aligned}
$$

which as we mentioned has a matter sector combination of real linear superfield $L$ and vector superfield $V$ that reproduce the Green-Schwarz mechanism in supergravity [36]. Here

$$
W_{\alpha}(V)=-\frac{1}{4} \bar{\nabla}^{2} \nabla_{\alpha} V
$$

is the standard field strength chiral superfield. For the pure gauge sector we have the bosonic components

$$
\frac{1}{4} \int d^{2} \theta \mathcal{E} W^{2}(V)+c . c .=-\frac{1}{4} e F^{m n} F_{m n}(v)+\frac{1}{2} e \mathrm{D}^{2}
$$

for

$$
F_{m n}(v)=\partial_{m} v_{n}-\partial_{n} v_{m}
$$

Now we can find the full bosonic sector of (2.11)

$$
\begin{aligned}
e^{-1} \mathcal{L}^{B}= & \frac{1}{2} M_{P}^{2}\left(R+6 H_{m} H^{m}\right)+2 M_{P}^{2} A_{m}^{-} H^{m} \\
& -\frac{1}{2} \partial \phi \partial \phi+\frac{1}{2} h_{m} h^{m}+\frac{1}{2} \mathrm{D}^{2}-\frac{1}{4} F^{m n} F_{m n} \\
& +\frac{1}{2} g M v_{m}\left(h^{m}+2 \phi H^{m}\right)-\frac{1}{2} g M \phi \mathrm{D} .
\end{aligned}
$$


Let us integrate out the supergravity auxiliary and the $h$-field. First we make $h$ and $H$ unconstrained by introducing Lagrange multipliers $X$ and $Y$

$$
\begin{aligned}
e^{-1} \mathcal{L}_{\text {aux }}= & 3 M_{P}^{2} H_{m} H^{m}+2 M_{P}^{2} A_{m}^{-} H^{m}+\partial_{n} X H^{n} \\
& -\frac{1}{2} g M \phi \mathrm{D}+\frac{1}{2} g M v_{m}\left(h^{m}+2 \phi H^{m}\right) \\
& +\frac{1}{2} \mathrm{D}^{2}+\frac{1}{2} h_{m} h^{m}+\partial_{m} Y\left(h^{m}+2 \phi H^{m}\right) .
\end{aligned}
$$

From the equations of motion of $A_{m}^{-}$we find the condition

$$
H^{m}=0
$$

and from the $H_{m}$ equations we find

$$
6 M_{P}^{2} H_{m}+2 M_{P}^{2} A_{m}^{-}+g M v_{m} \phi+\partial_{m} X+2 \phi \partial_{m} Y=0
$$

which combined with (2.17) leads to

$$
A_{m}^{-}=-\frac{g M v_{m} \phi+\partial_{m} X+2 \phi \partial_{m} Y}{\left(2 M_{P}^{2}\right)} .
$$

In fact the equation (2.19) never shows up, except in the supersymmetry transformations of the on-shell theory. The auxiliary Lagrangian becomes

$$
e^{-1} \mathcal{L}_{\text {aux }}=-\frac{1}{2} g M \phi \mathrm{D}+\frac{1}{2} g M v_{m} h^{m}+\frac{1}{2} \mathrm{D}^{2}+\frac{1}{2} h_{m} h^{m}+\partial_{m} Y h^{m} .
$$

Integrating out $h^{m}$ and D we find

$$
e^{-1} \mathcal{L}_{\text {aux }}=-\frac{1}{8}\left(g M v_{m}+2 \partial_{m} Y\right)^{2}-\frac{g^{2}}{8} M^{2} \phi^{2} .
$$

The model (2.11) after integrating out all the non-propagating fields is

$$
e^{-1} \mathcal{L}^{B}=\frac{1}{2} M_{P}^{2} R-\frac{1}{2} \partial \phi \partial \phi-\frac{g^{2}}{8} M^{2} \phi^{2}-\frac{1}{4} F^{m n} F_{m n}(v)-\frac{1}{8} g^{2} M^{2} v^{m} v_{m}
$$

where we have shifted

$$
v_{m} \rightarrow v_{m}+\frac{2}{g M} \partial_{m} Y
$$

It is clear from (2.23) that the Stueckelberg mechanism is at work.

The sector relevant to inflation reads

$$
e^{-1} \mathcal{L}_{\text {scalar }}=\frac{1}{2} M_{P}^{2} R-\frac{1}{2} \partial \phi \partial \phi-\frac{m^{2}}{2} \phi^{2}
$$

where we have replaced

$$
g M=2 m
$$

which is fixed by the observational data (see for example [10]) to be

$$
m \sim 10^{13} \mathrm{GeV} .
$$


During inflation since the $\eta$ slow-roll parameter is small we see that indeed

$$
\frac{g M}{H} \sim \frac{M_{P}}{\phi} \ll 1
$$

and relation (2.8) holds.

We see that the model (2.11) successfully reproduces the simplest model of chaotic inflation (1.2). Moreover there is no ambiguity in choosing the inflaton field, which is a common issue in supergravity inflation. The ambiguity is resolved by the simple fact that there is no other scalar in the first place. Indeed, thanks to the Stueckelberg mechanism the second scalar of the inflaton multiplet is eaten by the vector field. Thus we have a model of single-field chaotic inflation in supergravity which is technically natural employing a softly broken shift symmetry.

We should mention that single-field inflationary models with the use of massive vector multiplets have been introduced only recently in the literature [23-25], and in particular in ref. [24] a very interesting discussion on their properties can be found, including a realization of the chaotic model. In fact the model studied here is dual to the chaotic models of ref. [24], and thus essentially reproduces equivalent results. On the other hand the "linear superfield - vector superfield" picture we presented makes the technical naturalness of the model manifest in the new-minimal supergravity formulation.

As we have mentioned earlier, the Lagrangian (2.11) describes a massive vector multiplet. This may be seen by rewriting (2.11) as

$$
\begin{aligned}
\mathcal{L}= & -2 M_{P}^{2} \int d^{4} \theta E V_{\mathrm{R}}-g M \int d^{4} \theta E L\left(V+\frac{1}{g M} \Phi+\frac{1}{g M} \bar{\Phi}\right) \\
& +\frac{1}{4}\left[\int d^{2} \theta \mathcal{E} W^{2}(V)+\text { h.c. }\right]-\int d^{4} \theta E L^{2}
\end{aligned}
$$

where now $L$ is unconstrained. From (2.28) we see that the chiral superfield $\Phi$ has to carry a vanishing $\mathrm{R}$-charge for the chiral-linear duality to be possible. Then by integrating out $L$ from (2.28) we have

$$
\mathcal{L}=-2 M_{P}^{2} \int d^{4} \theta E V_{\mathrm{R}}+\frac{1}{4}\left[\int d^{2} \theta \mathcal{E} W^{2}(V)+\text { h.c. }\right]+\frac{1}{4} g^{2} M^{2} \int d^{4} \theta E V^{2}
$$

where we have shifted

$$
V \rightarrow V-\frac{1}{g M} \Phi-\frac{1}{g M} \bar{\Phi}
$$

The Lagrangian (2.29) describes a massive vector multiplet coupled to the new-minimal supergravity. It is easy to see that the bosonic sector of (2.29) will be given again by (2.22). In the limit $g M \rightarrow 0$ gauge invariance is restored, and the theory will be described by a massless vector multiplet and a massless chiral multiplet. Indeed in this limit the theory will become

$$
\mathcal{L}=-2 M_{P}^{2} \int d^{4} \theta E V_{\mathrm{R}}+\frac{1}{4}\left[\int d^{2} \theta \mathcal{E} W^{2}(V)+\text { h.c. }\right]+\frac{1}{2} \int d^{4} \theta E \bar{\Phi} \Phi
$$


and the shift symmetry will translate into

$$
\Phi \rightarrow \Phi+d M_{P}
$$

In (2.32) the constant $d$ can be complex. Note that (2.28) allowed only for a pure imaginary shift of the chiral superfield and thus only in the $g M \rightarrow 0$ limit $d$ can be complex. The fact that the shift (2.32) is a symmetry of (2.31) is connected to the structure of the new-minimal supergravity, which gives

$$
\int d^{4} \theta E \Phi=\int d^{4} \theta E \bar{\Phi}=0
$$

for a chiral superfield with vanishing R-charge.

If we instead start with the most general (up to two derivatives), gauge invariant coupling of the real linear with the vector multiplet, the superspace Lagrangian reads

$$
\begin{aligned}
\mathcal{L}= & -2 M_{P}^{2} \int d^{4} \theta E V_{\mathrm{R}}+\frac{1}{4}\left[\int d^{2} \theta \mathcal{E} W^{2}(V)+\text { h.c. }\right] \\
& -g M \int d^{4} \theta E L V-\int d^{4} \theta E \mathcal{F}(L)
\end{aligned}
$$

and after integrating out all the auxiliary field sector we find the bosonic part

$$
e^{-1} \mathcal{L}^{B}=\frac{1}{2} M_{P}^{2} R-\frac{1}{4} \mathcal{F}^{\prime \prime} \partial \phi \partial \phi-\frac{g^{2}}{8} M^{2} \phi^{2}-\frac{1}{4} F^{m n} F_{m n}(v)-\frac{1}{4 \mathcal{F}^{\prime \prime}} g^{2} M^{2} v^{m} v_{m}
$$

where

$$
\mathcal{F}^{\prime \prime}(\phi)=\frac{\partial^{2} \mathcal{F}(\phi)}{\partial \phi \partial \phi}
$$

Again in (2.35) the vector has eaten the Lagrange multiplier $Y$.

For a general kinematic function

$$
\mathcal{F}(\phi)=c_{0}+c_{2} \phi^{2}+c_{3} \phi^{3}+\ldots
$$

when all the higher order terms are present there is no shift symmetry, and in general $\mathcal{F}(\phi)$ will receive large corrections. Only in the case when

$$
c_{n}=0, \quad n \geq 3
$$

does $\mathcal{F}$ respect the shift symmetry and any correction to the higher order terms will have to be generated by the Green-Schwarz term and thus be suppressed.

\section{Chaotic inflation in old-minimal supergravity}

Now we investigate the embedding of the quadratic chaotic model in the old-minimal superspace [48]. This supergravity also originates from the superconformal supergravity after appropriate gauge fixing [39-43]. A complete treatment of the curvature superfields of this theory can be found in ref. [49]. In addition to the graviton and the gravitino, the pure theory contains two auxiliary fields: a complex scalar $u$, and a real vector $b_{m}$. It is 
well known how to couple a self-interacting massive vector multiplet to the old-minimal supergravity $[20,21]$.

Following the results of the previous section, we consider the superspace Lagrangian in the old-minimal formulation

$$
\begin{aligned}
\mathcal{L}= & -3 M_{P}^{2} \int d^{2} \Theta 2 \mathcal{E} \mathcal{R}+\text { h.c. }+\frac{1}{4}\left[\int d^{2} \Theta 2 \mathcal{E} W^{2}(V)+\text { h.c. }\right] \\
& -g M \int d^{4} \theta E \mathcal{Q} V-\int d^{4} \theta E \mathcal{G}(\mathcal{Q})
\end{aligned}
$$

where $\mathcal{Q}$ is a real linear multiplet with definition [50]

$$
\left(\overline{\mathcal{D}}^{2}-8 \mathcal{R}\right) \mathcal{Q}=0
$$

We see from (3.2) that the shift symmetry argument used in the new-minimal case does not apply, since the shift

$$
\mathcal{Q} \rightarrow \mathcal{Q}+c M_{P}
$$

for a real constant $c$, violates the definition (3.2). Thus a choice of a quadratic kinematic function

$$
\mathcal{G}(\mathcal{Q})=\mathcal{Q}^{2}
$$

is not protected by a shift symmetry.

To find the component form and relate to the known results, it is better to rewrite the Lagrangian (3.1) as the coupling of a massive vector multiplet to supergravity. For the part containing the real linear superfield we have

$$
\begin{aligned}
\mathcal{L}_{\mathcal{Q}} & =-g M \int d^{4} \theta E \mathcal{Q} V-\int d^{4} \theta E \mathcal{G}(\mathcal{Q}) \\
& =-g M \int d^{4} \theta E \mathcal{Q}\left(V+\frac{\Phi}{g M}+\frac{\bar{\Phi}}{g M}\right)-\int d^{4} \theta E \mathcal{G}(\mathcal{Q})
\end{aligned}
$$

In (3.5) the superfield $\mathcal{Q}$ is unconstrained, and it may be integrated out via the equations of motion

$$
\mathcal{G}^{\prime}(\mathcal{Q})=-g M\left(V+\frac{\Phi}{g M}+\frac{\bar{\Phi}}{g M}\right)
$$

The theory then becomes

$$
\mathcal{L}=\frac{1}{4} \int d^{2} \Theta 2 \mathcal{E} W^{2}(V)+\text { c.c. }+\int d^{2} \Theta 2 \mathcal{E}\left\{-\frac{1}{8}\left(\overline{\mathcal{D}}^{2}-8 \mathcal{R}\right) \mathcal{Z}(V)\right\}+\text { c.c. }
$$

with

$$
\mathcal{Z}(V)=-3 M_{P}^{2}-[\mathcal{G}(\mathcal{Q})+g M \mathcal{Q} V]_{\mathcal{G}^{\prime}(\mathcal{Q})=-g M V} .
$$

The Lagrangian (3.7) is defined for any hermitian function $\mathcal{Z}(V)$ of dimension $[\mathcal{Z}(V)]=2$ of the real vector superfield $V$. 
To turn to component form let us first define the components of the massive vector multiplet as

$$
\begin{aligned}
C & =V \mid \\
N & =-\frac{1}{4} \mathcal{D}^{2} V \mid \\
v_{\alpha \dot{\alpha}} & =-\frac{1}{2}\left[\mathcal{D}_{\alpha}, \overline{\mathcal{D}}_{\dot{\alpha}}\right] V \mid \\
\mathrm{D} & =\frac{1}{8} \mathcal{D}^{\alpha}\left(\overline{\mathcal{D}}^{2}-8 \mathcal{R}\right) \mathcal{D}_{\alpha} V \mid .
\end{aligned}
$$

The bosonic sector of (3.7) reads

$$
\begin{aligned}
e^{-1} \mathcal{L}^{B}= & -\frac{1}{4} F^{m n} F_{m n}(v)+\frac{1}{2} \mathrm{D}^{2}-\frac{1}{4} \mathcal{Z}^{\prime \prime} b^{m} b_{m}-\frac{1}{3} \mathcal{Z}^{\prime} \bar{u} \bar{N}-\frac{1}{3} \mathcal{Z}^{\prime} u N+\frac{1}{9} \mathcal{Z} u \bar{u} \\
& +\frac{1}{6} \mathcal{Z} R+\mathcal{Z}^{\prime \prime} N \bar{N}-\frac{1}{9} \mathcal{Z} b^{m} b_{m}+\frac{1}{2} \mathcal{Z}^{\prime} \mathrm{D}-\frac{1}{4} \mathcal{Z}^{\prime \prime} \partial C \partial C+\frac{1}{3} \mathcal{Z}^{\prime} b^{m} v_{m}
\end{aligned}
$$

where now $\mathcal{Z}$ is a function of the lowest component $C$ and

$$
\mathcal{Z}^{\prime}=\frac{\partial \mathcal{Z}}{\partial C}, \quad \mathcal{Z}^{\prime \prime}=\frac{\partial^{2} \mathcal{Z}}{\partial C \partial C} .
$$

After integrating out the auxiliary sector and performing the appropriate Weyl rescalings one finds [21]

$$
\begin{aligned}
e^{-1} \mathcal{L}^{B}= & \frac{1}{2} M_{P}^{2} R+\frac{1}{2} M_{P}^{2} \mathcal{J}^{\prime \prime} \partial C \partial C-\frac{1}{2} M_{P}^{4}\left(\mathcal{J}^{\prime}\right)^{2} \\
& -\frac{1}{4} F^{m n} F_{m n}(v)+\frac{1}{2} M_{P}^{2} \mathcal{J}^{\prime \prime} v^{m} v_{m}
\end{aligned}
$$

where

$$
\mathcal{J}(C)=\frac{3}{2} \ln \left[-\frac{1}{3 M_{P}^{2}} \mathcal{Z}(C)\right] .
$$

For a ghost-free theory one should have

$$
\mathcal{J}^{\prime \prime}<0 \text {. }
$$

It can be easily seen that the $\mathcal{J}(C)$ which reproduces the quadratic chaotic model is given by

$$
\mathcal{J}=-\frac{m^{2}}{2 M_{P}^{2}} C^{2}
$$

and the part of (3.12) relevant to inflation will read

$$
e^{-1} \mathcal{L}_{\text {scalar }}=\frac{1}{2} M_{P}^{2} R-\frac{1}{2} \partial \psi \partial \psi-\frac{m^{2}}{2} \psi^{2}
$$

for the inflaton

$$
\psi=m C .
$$

Again this is a single-field inflationary model. 
Nevertheless the function (3.15) does not correspond to a vector superfield self-coupling of the form

$$
-\frac{1}{2} m^{2} \int d^{4} \theta E V^{2}
$$

but will come from a more involved function of $V$. Indeed, the vector superfield function inside (3.7) has to have the form

$$
\mathcal{Z}(V)=-3 M_{P}^{2} e^{-\frac{m^{2}}{3 M_{P}^{2}} V^{2}}
$$

which leads to (3.15) and (3.16). On the other hand, a quadratic term as (3.18) will give rise to an exponential potential. In fact the coupling of the massive vector multiplet to standard supergravity was investigated in ref. [24] reproducing, among other models, also quadratic chaotic inflation.

Let us recapitulate for a moment. We have shown that starting with a theory of quadratic chaotic inflation we can embed it in supergravity in two distinct ways. In one case (the new-minimal) there is a softly broken shift symmetry in superspace which can be used to give a technical naturalness argument against higher order corrections. The embedding can be also straightforwardly carried out in the old-minimal formulation, but in this case there is no obvious way to render the theory technically natural.

Finally, one may also interpret (3.7) as a supergravity model with a gauged chiral sector [48] of the form

$$
\begin{aligned}
\mathcal{L}= & \int d^{2} \Theta 2 \mathcal{E}\left\{-\frac{1}{8}\left(\overline{\mathcal{D}}^{2}-8 \mathcal{R}\right) \mathcal{Z}\left(\ln \left[\bar{\Phi} e^{V} \Phi\right]\right)\right\}+\text { c.c. } \\
& +\frac{1}{4} \int d^{2} \Theta 2 \mathcal{E} W^{2}(V)+\text { c.c. }
\end{aligned}
$$

where the real part of the lowest component of the chiral superfield

$$
S=\ln \Phi
$$

will drive inflation, while the imaginary will be eaten by the massive vector.

\section{Conclusions}

We have studied the embedding of the quadratic model of chaotic inflation into the newminimal and the old-minimal theories of supergravity, with the use of massive vector multiplets. This embedding is quite straightforward and reproduces a single-field inflation model. This stems from the underlying Stueckelberg (or BEH) mechanism, where the second unwanted component of the scalar multiplet is eaten by the massive vector. Indeed this new mechanism has been investigated previously in a series of papers [23-25]. The simplicity and generality of the models indicate that their detailed cosmological properties deserve further study, a problem to which we will return in the future.

Our additional interest here was the notion of technical naturalness in superspace. As we have demonstrated, the model in the new-minimal formulation naturally evades the $\eta$ problem, due to a softly broken superspace shift symmetry of the real linear superfield. In 
the old-minimal formulation, even though the quadratic chaotic model can be successfully embedded, we could not identify a corresponding mechanism.

Closing, let us make a final comment on the 4D Green-Schwarz terms. These were merely introduced to generate a potential for the inflaton, and not to cancel some anomaly. Nevertheless, the specific combination of couplings we have used exactly reproduces the $4 \mathrm{D}$ analog of the Green-Schwarz mechanism, which leads one to hope that it would be possible to embed the theory into a UV complete superstring model. However, in order to protect the form of the potential it is essential that the model exhibits the shift symmetry. If such a model existed one could extend the technical naturalness of this paper to a topdown naturalness. The identification of specific superstring sectors with the supergravity properties discussed in this article would offer a new insight to string inflation which we leave for future research.

\section{Acknowledgments}

We thank I. Dalianis and A. Kehagias for discussion and correspondence. We thank R. Kallosh, A. Linde and M. Porrati for comments on the first version. This work is supported by the Grant agency of the Czech republic under the grant P201/12/G028.

Open Access. This article is distributed under the terms of the Creative Commons Attribution License (CC-BY 4.0), which permits any use, distribution and reproduction in any medium, provided the original author(s) and source are credited.

\section{References}

[1] BICEP2 collaboration, P.A.R. Ade et al., Detection of B-mode polarization at degree angular scales by BICEP2, Phys. Rev. Lett. 112 (2014) 241101 [arXiv: 1403.3985] [INSPIRE].

[2] A. Kehagias and A. Riotto, Remarks about the tensor mode detection by the BICEP2 collaboration and the super-Planckian excursions of the inflaton field, Phys. Rev. D 89 (2014) 101301 [arXiv: 1403.4811] [INSPIRE].

[3] L.E. Ibáñez and I. Valenzuela, BICEP2, the Higgs mass and the SUSY-breaking scale, arXiv:1403.6081 [INSPIRE].

[4] G. Dvali and C. Gomez, BICEP2 in corpuscular description of inflation, arXiv:1403.6850 [INSPIRE].

[5] D.H. Lyth, BICEP2, the curvature perturbation and supersymmetry, arXiv:1403.7323 [INSPIRE].

[6] N. Kaloper and L. Sorbo, A natural framework for chaotic inflation, Phys. Rev. Lett. 102 (2009) 121301 [arXiv:0811.1989] [INSPIRE].

[7] N. Kaloper and A. Lawrence, Natural chaotic inflation and UV sensitivity, Phys. Rev. D 90 (2014) 023506 [arXiv:1404.2912] [INSPIRE].

[8] Planck collaboration, P.A.R. Ade et al., Planck 2013 results. XXII. Constraints on inflation, arXiv:1303.5082 [INSPIRE].

[9] A.D. Linde, Chaotic inflation, Phys. Lett. B 129 (1983) 177 [InSPIRE]. 
[10] D.H. Lyth and A. Riotto, Particle physics models of inflation and the cosmological density perturbation, Phys. Rept. 314 (1999) 1 [hep-ph/9807278] [INSPIRE].

[11] A.D. Linde, Inflationary cosmology, Lect. Notes Phys. 738 (2008) 1 [arXiv:0705. 0164] [INSPIRE].

[12] M. Kawasaki, M. Yamaguchi and T. Yanagida, Natural chaotic inflation in supergravity, Phys. Rev. Lett. 85 (2000) 3572 [hep-ph/0004243] [INSPIRE].

[13] V. Demozzi, A. Linde and V. Mukhanov, Supercurvaton, JCAP 04 (2011) 013 [arXiv: 1012.0549] [INSPIRE].

[14] R. Kallosh and A. Linde, New models of chaotic inflation in supergravity, JCAP 11 (2010) 011 [arXiv: 1008.3375] [INSPIRE].

[15] R. Kallosh, A. Linde and T. Rube, General inflaton potentials in supergravity, Phys. Rev. D 83 (2011) 043507 [arXiv: 1011.5945] [InSPIRE].

[16] S. Ferrara, A. Kehagias and A. Riotto, The imaginary Starobinsky model, Fortsch. Phys. 62 (2014) 573 [arXiv: 1403. 5531] [InSPIRE].

[17] R. Kallosh, A. Linde, B. Vercnocke and W. Chemissany, Is imaginary Starobinsky model real?, JCAP 07 (2014) 053 [arXiv: 1403.7189] [INSPIRE].

[18] J. Ellis, M.A.G. García, D.V. Nanopoulos and K.A. Olive, Resurrecting quadratic inflation in no-scale supergravity in light of BICEP2, JCAP 05 (2014) 037 [arXiv:1403.7518] [INSPIRE].

[19] K. Hamaguchi, T. Moroi and T. Terada, Complexified Starobinsky inflation in supergravity in the light of recent BICEP2 result, Phys. Lett. B 733 (2014) 305 [arXiv:1403.7521] [INSPIRE].

[20] S. Mukhi, Massive vector multiplet coupled to supergravity, Phys. Rev. D 20 (1979) 1839 [INSPIRE].

[21] A. Van Proeyen, Massive vector multiplets in supergravity, Nucl. Phys. B 162 (1980) 376 [INSPIRE].

[22] S. Cecotti, S. Ferrara, M. Porrati and S. Sabharwal, New minimal higher derivative supergravity coupled to matter, Nucl. Phys. B 306 (1988) 160 [InSPIRE].

[23] F. Farakos, A. Kehagias and A. Riotto, On the Starobinsky model of inflation from supergravity, Nucl. Phys. B 876 (2013) 187 [arXiv:1307.1137] [INSPIRE].

[24] S. Ferrara, R. Kallosh, A. Linde and M. Porrati, Minimal supergravity models of inflation, Phys. Rev. D 88 (2013) 085038 [arXiv: 1307.7696] [InSPIRE].

[25] S. Ferrara, R. Kallosh, A. Linde and M. Porrati, Higher order corrections in minimal supergravity models of inflation, JCAP 11 (2013) 046 [arXiv:1309.1085] [INSPIRE].

[26] S. Ferrara, P. Fré and A.S. Sorin, On the topology of the inflaton field in minimal supergravity models, JHEP 04 (2014) 095 [arXiv: 1311.5059] [INSPIRE].

[27] S. Ferrara, P. Fré and A.S. Sorin, On the gauged Kähler isometry in minimal supergravity models of inflation, Fortsch. Phys. 62 (2014) 277 [arXiv:1401.1201] [INSPIRE].

[28] K. Kadota and M. Yamaguchi, D-term chaotic inflation in supergravity, Phys. Rev. D 76 (2007) 103522 [arXiv:0706.2676] [InSPIRE].

[29] K. Kadota, T. Kawano and M. Yamaguchi, New D-term chaotic inflation in supergravity and leptogenesis, Phys. Rev. D 77 (2008) 123516 [arXiv:0802.0525] [INSPIRE].

[30] I. Dalianis and F. Farakos, Higher derivative D-term inflation in new-minimal supergravity, arXiv:1403.3053 [INSPIRE]. 
[31] G. 't Hooft, Naturalness, chiral symmetry, and spontaneous chiral symmetry breaking, NATO Adv. Study Inst. Ser. B Phys. 59 (1980) 135 [inSPIRE].

[32] M.F. Sohnius and P.C. West, An alternative minimal off-shell version of $N=1$ supergravity, Phys. Lett. B 105 (1981) 353 [inSPIRE].

[33] M. Sohnius and P.C. West, The tensor calculus and matter coupling of the alternative minimal auxiliary field formulation of $N=1$ supergravity, Nucl. Phys. B 198 (1982) 493 [INSPIRE].

[34] S. Ferrara and S. Sabharwal, Structure of new minimal supergravity, Annals Phys. 189 (1989) 318 [inSPIRE].

[35] B.A. Ovrut and C. Schwiebert, Linear multiplets coupled to new minimal supergravity, Nucl. Phys. B 321 (1989) 163 [InSPIRE].

[36] S. Cecotti, S. Ferrara and L. Girardello, Massive vector multiplets from superstrings, Nucl. Phys. B 294 (1987) 537 [InSPIRE].

[37] G. Lopes Cardoso and B.A. Ovrut, A Green-Schwarz mechanism for D $=4, N=1$ supergravity anomalies, Nucl. Phys. B 369 (1992) 351 [INSPIRE].

[38] W. Siegel, Gauge spinor superfield as a scalar multiplet, Phys. Lett. B 85 (1979) 333 [INSPIRE].

[39] T. Kugo and S. Uehara, Conformal and Poincaré tensor calculi in $N=1$ supergravity, Nucl. Phys. B 226 (1983) 49 [inSPIRE].

[40] S. Ferrara, L. Girardello, T. Kugo and A. Van Proeyen, Relation between different auxiliary field formulations of $N=1$ supergravity coupled to matter, Nucl. Phys. B 223 (1983) 191 [INSPIRE].

[41] S.J. Gates, M.T. Grisaru, M. Roček and W. Siegel, Superspace or one thousand and one lessons in supersymmetry, hep-th/0108200 [INSPIRE].

[42] I.L. Buchbinder and S.M. Kuzenko, Ideas and methods of supersymmetry and supergravity: a walk through superspace, IOP, Bristol U.K. (1995).

[43] D. Butter, $N=1$ conformal superspace in four dimensions, Annals Phys. 325 (2010) 1026 [arXiv:0906.4399] [INSPIRE].

[44] S. Ferrara, S. Sabharwal and M. Villasante, Curvatures and Gauss-Bonnet theorem in new minimal supergravity, Phys. Lett. B 205 (1988) 302 [INSPIRE].

[45] S. Cecotti, S. Ferrara, L. Girardello, A. Pasquinucci and M. Porrati, Matter coupled supergravity with Gauss-Bonnet invariants: component Lagrangian and supersymmetry breaking, Int. J. Mod. Phys. A 3 (1988) 1675 [inSPIRE].

[46] B.A. Ovrut and S. Kalyana Rama, New minimal supergravity, Chern-Simons terms and induced massive gauge fields, Nucl. Phys. B 333 (1990) 380 [InSPIRE].

[47] B.A. Ovrut and S. Kalyana Rama, Lorentz and U(1) Chern-Simons terms in new minimal supergravity, Phys. Lett. B 254 (1991) 132 [INSPIRE].

[48] J. Wess and J. Bagger, Supersymmetry and supergravity, Princeton Univ. Pr., Princeton U.S.A. (1992).

[49] S. Ferrara and M. Villasante, Curvatures, Gauss-Bonnet and Chern-Simons multiplets in old minimal $N=1$ supergravity, J. Math. Phys. 30 (1989) 104 [InSPIRE].

[50] P. Binetruy, G. Girardi and R. Grimm, Supergravity couplings: a geometric formulation, Phys. Rept. 343 (2001) 255 [hep-th/0005225] [INSPIRE]. 